\title{
The Pompeiu problem
}

\author{
A. G. Ramm \\ Mathematics Department, Kansas State University, Manhattan, KS 66506, USA \\ Email:ramm@math.ksu.edu
}

\section{Abstract}

Let $f \in L_{\text {loc }}^{1}\left(\mathbb{R}^{n}\right) \cap \mathcal{S}^{\prime}$, where $\mathcal{S}^{\prime}$ is the Schwartz class of distributions. Assume that

$$
\int_{\sigma(D)} f(x) d x=0 \quad \forall \sigma \in G,
$$

where $D \subset \mathbb{R}^{n}, n \geq 2$, is a bounded domain, the closure $\bar{D}$ of which is $C^{1}$-diffeomorphic to a closed ball. Then the complement of $\bar{D}$ is connected and path connected. Here $G$ denotes the group of all rigid motions in $\mathbb{R}^{n}$. This group consists of all translations and rotations.

It is conjectured that if $f \neq 0$ and $(*)$ holds, then $D$ is a ball. Other conjectures, equivalent to the above one, are formulated and discussed. Several new short proofs are given for various results. A new idea concerning the possible approach to the Pompeiu problem is formulated. The presentation is self-contained.

Keywords: Pompeiu problem, Symmetry problems.

\section{Introduction}

In this paper the problem known as the Pompeiu problem is formulated and discussed. This problem originated in Pompeiu's paper [9], of 1929. The problem in a modern formulation is stated below as Conjecture 1, and is still open.

Dimitrie Pompeiu (1873-1954) was born in Romania and got his Ph.D in 1905 at the Sorbonne, in Paris, under the direction of H. Poincaré. He is known mainly for the Pompeiu problem and for the Cauchy-Pompeiu formula in complex analysis.

Let us formulate the Pompeiu problem as it is understood today.

Let $f \in L_{l o c}^{1}\left(\mathbb{R}^{n}\right) \cap \mathcal{S}^{\prime}$, where $\mathcal{S}^{\prime}$ is the Schwartz class of distributions, and

$$
\int_{\sigma(D)} f(x) d x=0 \quad \forall \sigma \in G
$$

where $G$ is the group of all rigid motions of $\mathbb{R}^{n}$, consisting of all translations and rotations, and $D \subset \mathbb{R}^{n}$ is a bounded domain, the closure $\bar{D}$ of which is $C^{1}$-diffeomorphic to a closed ball. Under these assumptions the complement of $\bar{D}$ in $\mathbb{R}^{n}, n \geq 2$, is connected and path connected by the isotopy extension theorem, see [6].

The distribution space $\mathcal{S}^{\prime}$ in the assumption $f \in L_{l o c}^{1}\left(\mathbb{R}^{n}\right) \cap \mathcal{S}^{\prime}$ can be replaced without essential changes in the argument by the distribution space $\mathcal{D}^{\prime}$, where $\mathcal{D}$ is the space of $C_{0}^{\infty}\left(\mathbb{R}^{3}\right)$ functions.

In [9] the following question was raised:

Does (1) imply that $f=0$ ?

If yes, then we say that $D$ has $P$-property (Pompeiu's property), and write $D \in P$. Otherwise, we say that $D$ fails to have $P$-property, and write $D \in \bar{P}$. Pompeiu claimed in 1929 (see [9]) that every plane bounded domain has $P$-property. This claim turned out to be false: a counterexample was given 15 years later in [3]. The counterexample is a domain $D$ which is a disc, or a ball in $\mathbb{R}^{n}$ for $n>2$. If $D$ is a ball, then there are $f \neq 0$ for which equation 
(1) holds. The set of all $f \neq 0$, for which equation (1) holds, was constructed in [10]. There are infinitely many (a continuum) such $f$. Let us give the counterexample mentioned above.

Example 1. Suppose that $D \subset \mathbb{R}^{n}$ is a ball $B$ centered at the origin and of radius a. Then the Fourier transform of its characteristic function $\chi$ is (see, for instance, [12], Chapter 11):

$$
\tilde{\chi}(\xi)=\int_{B} e^{i \xi \cdot x} d x=(2 \pi a)^{n / 2} \frac{J_{n / 2}(a|\xi|)}{|\xi|^{n / 2}},
$$

where $J_{n / 2}$ is the Bessel function. It follows that if $|\xi|=s_{j, n} / a$, where $s_{j, n}$ is any positive zero of the Bessel function $J_{n / 2}(s)$, then $\tilde{\chi}(\xi)$ has a spherical set of zeros. This implies, as follows from Theorem 3, proved below, that there are $f \neq 0$ for which relation (1) holds.

A bibliography on the Pompeiu problem ( $P$-problem) can be found in [17] and in [10].

The current formulation of the $P$-problem is the following:

$P$-problem. Prove that if $D \subset \mathbb{R}^{n}$ is a bounded domain $C^{1}$-diffeomorphic to a ball and $D \in \bar{P}$, then $D$ is a ball.

We use the word ball also in the case $n=2$, when this word means disc, and discuss the $P$-problem in detail. This problem leads to some problems of general mathematical interest: a symmetry problem for partial differential equations, see Conjecture 2 below, and a problem in harmonic analysis, see Conjecture 3 below.

Let us make the following standing assumptions, that hold throughout this paper:

Assumptions $A$ :

$\left.A_{1}\right): D$ is a bounded domain, the closure of which is $C^{1}$-diffeomorphic to a closed ball, the boundary $S$ of $D$ is a closed connected $C^{1}$-smooth surface,

$\left.A_{2}\right)$ : $D$ fails to have P-property.

Our first conjecture is:

Conjecture 1. If Assumptions A hold, then $D$ is a ball.

In Section 2 this Conjecture is discussed. We prove that Conjecture 1 is equivalent to a symmetry problem for an over-determined boundary value problem for a partial differential equation. Namely, it is equivalent to the following conjecture.

Conjecture 2. If problem (3) (see below) has a solution, then $D$ is a ball.

Several symmetry problems were studied by the method similar to the one used in the proof of Theorem 1 , below, see also [13]-[15].

Conjectures 1 and 2 are equivalent to the following conjecture:

Conjecture 3. If Assumption $A_{1}$ holds and the Fourier transform $\tilde{\chi}_{D}$ of the characteristic function $\chi_{D}$ of the domain $D$ has a spherical surface of zeros, then $D$ is a ball.

Conjecture 3 can be generalized: the author thinks that if the Fourier transform $\tilde{\chi}_{D}$ of the characteristic function $\chi_{D}$ of the domain $D$ is spherically symmetric, that is, it is a function of $|\xi|$ only, then $D$ is a ball.

Three other conjectures are formulated in Sections 2 and 4.

The novel points in this paper include a proof of equivalence of Conjectures 1, 2 and 3, proofs of Theorems 1 , 2, 3, and Lemmas 1-5, and formulation of Conjecture 6 .

\section{Discussion of the Conjectures}

It is proved in [16] that if Assumptions A hold, then the boundary $S$ of $D$ is real-analytic. It is proved in Theorem 3 below, that if Assumptions A hold, then the problem

$$
\left(\nabla^{2}+k^{2}\right) u=1 \quad \text { in } \quad D,\left.\quad u\right|_{S}=0,\left.\quad u_{N}\right|_{S}=0, \quad k^{2}=\text { const }>0
$$

has a solution. In (3) $N=N_{s}$ is the outer unit normal to $S$ pointing out of $D, s \in S$ is a point on $S$.

Therefore, if (1) holds, then problem (3) has a solution.

Let us prove that if problem (3) has a solution, then $\tilde{\chi}_{D}$ has a spherical set of zeros, where $\chi_{D}$ is the characteristic function of the domain $D$. To prove this, let us multiply (3) by $e^{i k \alpha \cdot x}$, where $\alpha \in S^{n-1}$ is an arbitrary unit vector and $S^{n-1}$ is the unit sphere in $\mathbb{R}^{n}$, and integrate with respect to $x$ over $D$. Using an integration by parts and the boundary conditions (3) for $u$, one gets the desired relation:

$$
\tilde{\chi}_{D}(k \alpha)=0 \quad \forall \alpha \in S^{n-1} .
$$


Thus, $\tilde{\chi}_{D}$ has a spherical set of zeros.

Conjectures 1, 2, and 3 are equivalent in the sense that each of them claims that $D$ is a ball. But these three conjectures are also equivalent in the sense that if one of them is correct, then so are the remaining two.

Indeed, in the proof of Theorem 3 equation (3) was derived from equation (1), and the relation

$$
\tilde{\chi}_{D}(\xi)=0, \quad|\xi|=\text { const }>0,
$$

was derived from equation (3). From the proof of Theorem 3 it follows that equation (5) implies the equation

$$
\tilde{\chi}(\xi)=\left(\xi^{2}-k^{2}\right) \tilde{u}(\xi)
$$

(see equation (23) below, in the proof of Theorem 3), and the inverse Fourier transform of (6) yields equation (3) and the boundary conditions in (3). In this sense problem (3) is equivalent to relation (5), and Conjectures 2 and 3 are equivalent in the sense that if one of them is correct, so is the other one.

Furthermore, from the proof of Theorem 3 it follows that (5) implies (1). Indeed, equation (5) implies equation

$$
\tilde{f}(\xi) \overline{\tilde{\chi}\left(g^{-1} \xi\right)}=0
$$

(see equation (21) below), and the inverse Fourier transform of this equation yields relation (1).

In this sense relations (1), (3) and (5) are equivalent, and Conjectures 1, 2 and 3, are equivalent in the sense that if one of them is correct, so are the other two.

Problem (3) is an open symmetry problem of long standing for partial differential equations. Let us formulate another open symmetry problem for partial differential equations of long standing, known as M. Schiffer's conjecture (see, e.g., [4], [12]):

Conjecture 4. If the problem

$$
\left(\nabla^{2}+k^{2}\right) u=0 \quad \text { in } \quad D,\left.\quad u_{N}\right|_{S}=\text { const } \neq 0,\left.\quad u\right|_{S}=0, \quad k^{2}=\text { const }>0
$$

has a solution, then $D$ is a ball.

Note that Conjecture 2 can be formulated in the form similar to (8):

Conjecture 5. If the problem

$$
\left(\nabla^{2}+k^{2}\right) u=0 \quad \text { in } \quad D,\left.\quad u_{N}\right|_{S}=0,\left.\quad u\right|_{S}=\text { const } \neq 0, \quad k^{2}=\text { const }>0
$$

has a solution, then $D$ is a ball.

Lemma 0. Conjecture 5 is equivalent to Conjecture 2.

Proof. If (3) holds, then one can look for the solution $u$ of the form $u=v+c$, where $c$ is a constant. The boundary conditions in (3) imply $v=-c$ on $S$, and $v_{N}=0$ on $S$. Let us choose $c=1 / k^{2}$. Then the differential equation (3) implies

$$
\left(\nabla^{2}+k^{2}\right) v=1-k^{2} c=0 .
$$

Therefore, $v$ solves problem (9). Conversely, if $v$ solves problem (9), then $u=v+c$ solves (3) if $c$ is a suitable constant.

Conjecture 5 apparently is not equivalent to Conjecture 4, as follows from its formulation. These two conjectures are independent and long-standing.

The results, on which our discussion of Conjectures 1,2 and 3 is based, are Theorems 1, 2 and 3 . Theorems 1 and 2 were proved originally in [11], [10], and in the book [12], Chapter 11. A result, equivalent to Theorem 3 , had been proved originally in the paper [2] by a considerably longer and more complicated argument. Our proof follows the one in [12], Chapter 11. In the paper [8] the null-varieties of the Fourier transform of the characteristic function of a bounded domain $D$ are studied. The properties of these varieties and the geometrical properties of $D$ are related, of course, but it is not clear in what way they are related. Conjecture 3, if it is proved, is an interesting example of such a relation.

In Section 3 a relation of the Pompeiu problem in $\mathbb{R}^{2}$ to analyticity of $f$ is discussed. It is proved that if the domain $D \in P, f \in L_{l o c}^{1}\left(\mathbb{R}^{2}\right)$, and if $\int_{\partial \sigma(D)} f d z=0 \quad \forall \sigma \in G$, then $f$ is an entire function. An earlier discussion of this result can be found in [18]. We give a new short proof of a result from [18].

In Section 4 a new approach to the Pompeiu problem is outlined and a new conjecture is formulated.

To make our presentation essentially self-contained, proofs of Theorems 1, 2 and 3, are included in this paper.

Theorem 1. If Assumptions A hold, then

$$
[s, N]=u_{N}, \quad \forall s \in S
$$


where $[s, N]$ is the cross product in $\mathbb{R}^{3}$, and $u$ is a vector-function that solves the problem

$$
\left(\nabla^{2}+k^{2}\right) u=0 \quad \text { in } \quad D,\left.\quad u\right|_{S}=0 .
$$

If $n=2$, then $D$ is a plane domain, $S$ is a curve $C^{1}$-diffeomorphic to a circle, $u$ is a scalar solution to equation (11), and the boundary condition (10) yields $s_{1} N_{2}-s_{2} N_{1}=u_{N}, \quad \forall s \in S$, where $N_{j}, j=1,2$, are Cartesian coordinates of the unit normal $N$ to $S$ pointing out of $D$. Indeed, if $n=2$ then the cross product of two vectors $\left[s_{1} e_{1}+s_{2} e_{2}, N_{1} e_{1}+N_{2} e_{2}\right]$ is calculated by the formula $[s, N]=\left(s_{1} N_{2}-s_{2} N_{1}\right) e_{3}$, where $e_{3}$ is a unit vector, orthogonal to the plane domain $D$, and the triple $\left\{e_{j}\right\}_{j=1}^{3}$ is a standard orthonormal basis in $\mathbb{R}^{3}$.

Let us state the following new characterization of spheres. Several other characterizations of spheres are known. For example, if the mean curvature of a sufficiently smooth closed surface is a constant, then the surface is a sphere, see [1].

Theorem 2. If $S$ is a smooth surface homeomorphic to a sphere and $[s, N]=0$ on $S$, then $S$ is a sphere.

The proof of Theorem 2 will be given in the coordinate system in which the condition $[s, N]=0$ on $S$ is valid.

The following conclusion is an immediate consequence of Theorem 2 :

The conclusion of Conjecture 1 will be established if one proves, under the Assumptions A, that $[s, N]=0$ on $S$.

Let us start by proving Theorem 2, then Theorem 1 is proved, and, finally, we prove Theorem 3. It is assumed throughout, except in Section 3, that $n=3$. Our proofs of Theorems 1 and 3 can be used for any integer $n \geq 2$ without any essential changes. The proof of Theorem 2 uses the notion of the cross-product, and by this reason its proof should be modified for $n>3$.

Proof of Theorem 2. Let $n=3$ and assume that $s=s(p, q)$ is a parametric equation of the surface $S$. The normal $N$ to $S$ is a vector directed along the vector $\left[s_{p}, s_{q}\right]$, where $s_{p}$ denotes the partial derivative of the function $s(p, q)$ with respect to the parameter $p$. The assumption $[s, N]=0$ on $S$, yields

$$
\left[s,\left[s_{p}, s_{q}\right]\right]=s_{p} s \cdot s_{q}-s_{q} s \cdot s_{p}=0,
$$

where $s \cdot s_{q}$ is the dot product of two vectors in $\mathbb{R}^{3}$. At a non-singular point of $S$ the vectors $s_{p}$ and $s_{q}$ are linearly independent. The surface $S$ is smooth, so its points are non-singular. Therefore equation (12) implies $s \cdot s_{q}=0$ and $s \cdot s_{p}=0$, so

$$
\frac{\partial s \cdot s}{\partial p}=0, \quad \frac{\partial s \cdot s}{\partial q}=0 .
$$

Consequently,

$$
s \cdot s=\text { const } .
$$

This is an equation of a sphere in the coordinate system with the origin at the center of the sphere. Theorem 2 is proved.

Proof of Theorem 1. Let $n=3$ and $\mathcal{N}$ denote the set of all smooth solutions to equation (11) in a ball $B$, containing $D$, but these solutions do not necessarily satisfy the boundary condition (11). Assume that problem (3) has a solution. Multiply (3) by an arbitrary solution $h$ to equation (11) in a ball $B$, containing $D$, integrate by parts, take into account the boundary conditions in (3), and get the relation

$$
\int_{D} h(x) d x=0 \quad \forall h \in \mathcal{N} .
$$

The inclusion $h \in \mathcal{N}$ implies $\nabla h \in \mathcal{N}$, so

$$
\int_{D} \nabla h(x) d x=0 \quad \forall h \in \mathcal{N} .
$$

Since $h \in \mathcal{N}$ implies $h(g x) \in \mathcal{N}$ for all $g$, where $g$ is an arbitrary rotation in $\mathbb{R}^{3}$ about the origin $O$, one obtains

$$
\int_{D} h(g x) d x=0 \quad \forall h \in \mathcal{N}, \forall g .
$$

Let $O \in D$, and take an arbitrary straight line $\ell$ passing through $O$ and directed along a unit vector $\alpha$. Let $g=g(\phi)$ be the rotation about $\ell$ by an angle $\phi$ counterclockwise. Differentiate (15) with respect to $\phi$ and then set $\phi=0$, 
see [11]. A similar argument has been used in [13]-[15], where various symmetry problems have been studied. The result is

$$
\int_{D} \nabla h(x) \cdot[\alpha, x] d x=0
$$

where $[\alpha, x]$ is the cross product and · stands for the inner product in $\mathbb{R}^{3}$. Equation (16) is invariant with respect to translations because $\int_{D} \nabla h(x) d x=0 \forall h \in \mathcal{N}$. Using the relation $\nabla \cdot[\alpha, x]=0$, valid for any constant vector $\alpha$, one writes $\nabla h \cdot[\alpha, x]=\nabla \cdot h[\alpha, x]$, applies the divergence theorem and using the arbitrariness of $\alpha$ derives from (16) the following relation

$$
\int_{S} h(s)[s, N] d s=0, \quad \forall h \in \mathcal{N}
$$

which is also invariant with respect to translations. Indeed, $N=\left[s_{p}, s_{q}\right]$ and $\left[s_{p}, s_{q}\right]$ is invariant under translations. The integral $\int_{S} h(s)[\alpha, N] d s=0$ for any constant vector $\alpha$. This relation is invariant under translations because $\int_{S} h(s) N d s=\int_{D} \nabla h d x=0$, as was pointed out above.

Let us derive from (17) equation (10). To do this, we need the following result.

Lemma 1. The orthogonal complement in $L^{2}(S)$ of the set $M$ of the restrictions of all $h \in \mathcal{N}$ to $S$ is a finitedimensional space spanned by the functions $u_{j N}$, where $\left\{u_{j}\right\}_{j=1}^{J}$ is the basis of the eigenspace of the Dirichlet Laplacian in D, corresponding to the eigenvalue $k^{2}$.

Remark 1. From Lemmas 1 and 2 it follows that equation (17) implies equation (10). Indeed, it follows from equation (17) that $[s, N]$ is orthogonal in $L^{2}(S)$ to the set $M$. By Lemma 2, the set $M$ is dense in $L^{2}(S)$ in the set of all functions $f$ for which problem (18) is solvable. Therefore, by Lemma 1, each of the three components of the vector $[s, N]$ must be linear combinations of the functions $u_{j N}, 1 \leq j \leq J$. In other words, equation (10) holds.

Proof of Lemma 1. Note that the result of Lemma 1 is equivalent to the assertion that the boundary value problem

$$
\left(\nabla^{2}+k^{2}\right) h=0 \quad \text { in } \quad D,\left.\quad h\right|_{S}=f
$$

is solvable if and only if

$$
\int_{S} f u_{j N} d s=0, \quad 1 \leq j \leq J
$$

where $u_{j}, 1 \leq j \leq J$, is a basis of the solutions to problem (11).

The necessity of conditions (19) is proved by the relation

$$
0=\int_{D} u_{j}\left(\nabla^{2}+k^{2}\right) h d x=-\int_{S} f u_{j N} d s
$$

where an integration by parts and the boundary condition $u_{j}=0$ on $S$ were used, and equation (18) for $h$ was taken into account.

The sufficiency of conditions (19) is proved as follows. Denote by $H^{m}(D)$ the usual Sobolev spaces. Given an $f \in H^{3 / 2}(S)$, construct an arbitrary $F \in H^{2}(D)$, such that $\left.F\right|_{S}=\left.f\right|_{S}$. This is possible by the trace theorem. Define $w$ by the relation $w=h-F$. Then $w$ solves the problem

$$
\left(\nabla^{2}+k^{2}\right) w=-\left(\nabla^{2}+k^{2}\right) F \quad \text { in } \quad D,\left.\quad w\right|_{S}=0
$$

If such $w$ exists, that is, if problem (20) is solvable, then the function $h=w+F$ solves problem (18), and the sufficiency of condition (19) is proved. For the existence of $w$ it is necessary and sufficient that

$$
\int_{D}\left(\nabla^{2}+k^{2}\right) F u_{j} d x=0, \quad 1 \leq j \leq J .
$$

An integration by parts shows that these conditions are equivalent to conditions (19) because $u_{j}$ solve problem (11). Thus, Lemma 1 is proved.

Equation (17) says that $[s, N]$ is orthogonal to the set $M$, that is, to the restrictions of all $h \in \mathcal{N}$ to $S$.

Lemma 2. The set $M$ is dense in $L^{2}(S)$ in the set of all $f \in H^{3 / 2}(S)$ for which problem (18) is solvable. 
Proof of Lemma 2. Assume the contrary. Then for some $f \in H^{3 / 2}(S), f \neq 0$, problem (18) is solvable and

$$
\eta(y):=\int_{S} f(s) \psi(s, y) d s=0 \quad \forall y \in D^{\prime}:=\mathbb{R}^{3} \backslash D,
$$

where $\psi(x, y):=\frac{e^{i k|x-y|}}{4 \pi|x-y|} \in \mathcal{N}$ for $y \in B^{\prime}$, that is, outside a ball containing $D$. The function $\eta$ is a simple-layer potential which vanishes in $B^{\prime}$. Therefore, by the unique continuation property for solutions of the homogeneous Helmholtz equation, $\eta=0$ everywhere in $D^{\prime}$. Thus, it vanishes on $S$. Therefore, $\eta$ solves problem (11). By the jump relation for the normal derivative of $\eta$ across $S$, one has $f=\eta_{N}$, where $\eta_{N}$ is the limiting value of the normal derivative of $\eta$ on $S$ from inside $D$. If problem (18) is solvable, then, as we have proved, $f$ is orthogonal to all functions $u_{j N}$. The function $\eta_{N}$ is a linear combination of these functions. This and the relation $f=\eta_{N}$ prove that $f=0$. Consequently, we have proved the claimed density of $M$ in the set of all $H^{3 / 2}(S)$-functions $f$ for which problem (18) is solvable. Lemma 2 is proved.

This completes the proof of Theorem 2.

Theorem 3. Suppose Assumptions $A$ hold and relation (1) holds for some $f \neq 0$. Then problem (3) has a solution. Conversely, if problem (3) has a solution, then there exists $f \neq 0$ such that relation (1) holds.

Proof of Theorem 3. Write (1) as

$$
\int_{\mathbb{R}^{3}} f(g x+y) \chi(x) d x=0 \quad \forall y \in \mathbb{R}^{3}, \quad \forall g \in G,
$$

where $\chi(x)$ is the characteristic function of $D$. Applying the Fourier transform and the convolution theorem one gets

$$
\tilde{f}(\xi) \overline{\tilde{\chi}\left(g^{-1} \xi\right)}=0,
$$

where $\tilde{f}$ and $\tilde{\chi}$ are the Fourier transforms of $f$ and $\chi$, respectively, and the overbar stands for the complex conjugate. The Fourier transform of $f$ is understood in the sense of distributions. The Fourier transform $\tilde{\chi}$ is an entire function of exponential type because function $\chi$ has support $\bar{D}$, which is a bounded set. Moreover, $\tilde{\chi}$ is a uniformly bounded function of $\xi \in \mathbb{R}^{n}$. The product of the tempered distribution $\tilde{f}$ and the function $\tilde{\chi}$ is a tempered distribution also, that is, and element of $\mathcal{S}^{\prime}$.

Since $g^{-1}$ runs through all the rotations, one can replace $g^{-1}$ by $g$. It follows from (21) that

$$
\text { supp } \tilde{f}=\cup_{k} C_{k} \text {, where } C_{k}:=\left\{\xi: \tilde{\chi}(\xi)=0 \forall \xi: \xi^{2}-k^{2}=0\right\} .
$$

In other words, the support of the distribution $\tilde{f}$ is a subset of the union of spherical surfaces of zeros of $\tilde{\chi}$, the Fourier transform of the characteristic function of the bounded domain $D$. Since $\tilde{\chi}(\xi)$ is an entire function of exponential type, vanishing on an irreducible algebraic variety $\xi^{2}-k^{2}=0$ in $\mathbb{C}^{3}$, one concludes, using the division lemma, that

$$
\tilde{\chi}(\xi)=\left(\xi^{2}-k^{2}\right) \tilde{u}(\xi)
$$

where $\tilde{u}$ is an entire function of the same exponential type as $\tilde{\chi}$ ( see [5]). Therefore, by the Paley-Wiener theorem, the corresponding $u$ has compact support. Taking the inverse Fourier transform of equation (23), one gets:

$$
\left(-\nabla^{2}-k^{2}\right) u(x)=\chi(x) \quad \text { in } \quad \mathbb{R}^{3}, \quad u=0 \quad \text { if } \quad|x|>R,
$$

where $R>0$ is sufficiently large. By the elliptic regularity results, one concludes that $u \in H_{\text {loc }}^{2}\left(\mathbb{R}^{3}\right)$. Since $u$ solves the Helmholtz elliptic equation and vanishes near infinity, that is, in the region $|x|>R$, the uniqueness of the solution to the Cauchy problem to the equation (24) and the path connectedness of the complement $D_{1}:=\bar{D}^{\prime}$ of the closure $\bar{D}$ of $D$ allow one to conclude that $u=0$ in $D_{1}$. The connectedness and path connectedness of $D_{1}$ follow from our Assumptions A and from the isotopy extension theorem (see [6]). If $u=0$ in $D_{1}$ and $u \in H_{l o c}^{2}\left(\mathbb{R}^{3}\right)$, it follows from the Sobolev embedding theorem that the boundary conditions (3) hold. Since $\chi(x)=1$ in $D$, equation (3) holds. The converse statement in Theorem 3 has already been established above, in our discussion of the equivalence of Conjectures 1 and 2 . Theorem 3 is proved.

Let us prove the equivalence of Conjectures 1,2 and 3 in the following lemma. In the proof of this lemma we denote these conjectures I, II, and III. The argument, used in the proof of Theorem 3, is used in the proof of a part of Lemma 3.

Lemma 3. Conjectures 1,2 and 3 are equivalent. 
Proof of Lemma 3. III $\Rightarrow I I$. If an entire function of exponential type $\tilde{\chi}(\xi)$ vanishes on the irreducible algebraic manifold $\xi^{2}=k^{2}$, then the function $\tilde{u}:=\tilde{\chi}(\xi)\left(\xi^{2}-k^{2}\right)^{-1}$ is also entire and of the same exponential type. Its Fourier transform $u(x)$ solves problem $(3)$.

$I I \Rightarrow I I I$. If problem (3) has a solution, then multiply (3) by $e^{i k \alpha \cdot x}, \alpha \in S^{n-1}$, integrate over $D$, and then by parts, using the boundary conditions in (3) and the equation $\left(\nabla^{2}+k^{2}\right) e^{i k \alpha \cdot x}=0$. This yields (4).

$I \Rightarrow I I I$. Take the Fourier transform (in the distributional sense) of equation (1) and get $\overline{\tilde{\chi}\left(g^{-1} \xi\right)} \tilde{f}(\xi)=0$, where the overline stands for the complex conjugate. Therefore, supp $\tilde{f}=\cup_{k} C_{k}$, where $C_{k}=\left\{\xi: \xi^{2}=k^{2},\left.\tilde{\chi}\right|_{\xi^{2}=k^{2}}=0\right\}$, and the set $\{k\}$ is a discrete finite set of positive numbers. Thus, there is a $k>0$ such that (4) holds.

$I I I \Rightarrow I$. If (4) holds, then there exists an $\tilde{f} \neq 0$ supported on $C_{k}$. Then $\overline{\tilde{\chi}}\left(g^{-1} \xi\right) \tilde{f}(\xi)=0$. Taking the inverse Fourier transform of this relation yields (1).

Lemma 3 is proved

\section{Relation to analyticity}

The classical Morera theorem in complex analysis says that if $\int_{C} f(z) d z=0$ for any closed polygon $C$ in a domain $D$ of the complex plane, and if $f$ is continuously differentiable in $D$, then $f$ is analytic in $D$. A simple proof is based on a version of Green's formula:

$$
0=\int_{C} f(z) d z=2 i \int_{\Delta} \bar{\partial} f d x d y .
$$

Here $\Delta$ is the plane domain with the boundary $C$ and $\bar{\partial} f:=\frac{f_{x}+i f_{y}}{2}$. If $\int_{\Delta} \bar{\partial} f d x d y=0$ for any polygon $\Delta$, then one passes to the limit in the formula

$$
\frac{1}{|\Delta|} \int_{\Delta} \bar{\partial} f d x d y=0
$$

where $|\Delta|$ is the area of $\Delta$ and the limit is taken as diam $\Delta \rightarrow 0$, so that $\Delta$ shrinks uniformly in directions to a point $(x, y) \in \Delta$. Then for almost all points in $D$ one gets $\bar{\partial} f=0$, and if $\bar{\partial} f$ is continuous, then $\bar{\partial} f=0$ everywhere in $D$. This implies that $f$ is analytic in $D$.

One may ask if the assumption that $f$ is continuously differentiable can be replaced by a weaker assumption, and if the set of polygons can be replaced by some other sets. The answer to this first question is easy: if $f \in L_{l o c}^{1}(D)$, then one considers a mollified function $f_{\epsilon}(z):=\int_{\zeta:|z-\zeta| \leq \epsilon} \omega_{\epsilon}(z-\zeta) f(\zeta) d u d v$, where $\zeta=u+i v$ and $\omega_{\epsilon}(z)$ is the standard mollifying kernel ([7], p.14). It is known that $f_{\epsilon} \rightarrow f$ in $L^{1}(D)$ as $\epsilon \rightarrow 0$, and one can select a subsequence $\epsilon_{j} \rightarrow 0$, such that $f_{\epsilon_{j}} \rightarrow f$ almost everywhere in $D$. If $\int_{C} f(z) d z=0$ for any closed polygon $C$, then $\int_{C} f_{\epsilon}(z) d z=0$ for any closed polygon $C$, and the above argument, applied to the $C^{1}$ - smooth $f_{\epsilon}$, leads to the conclusion that $f_{\epsilon}$ is analytic in $D$ for all sufficiently small $\epsilon$. Since a sequence $f_{\epsilon_{j}}$ of analytic functions converges to $f$ in $L^{1}(D)$ and almost everywhere in $D$, one concludes that $f$ is analytic in $D$. This follows from the closedness of the differential operator $\bar{\partial}$. Namely, one has $\left\|f_{\epsilon_{j}}-f\right\|_{L^{1}(D)} \rightarrow 0$ and $\bar{\partial} f_{\epsilon_{j}}=0$, so $\left\|\bar{\partial} f_{\epsilon_{j}}-0\right\|_{L^{1}(D)} \rightarrow 0$. Consequently, $f$ belongs to the domain of the operator $\bar{\partial}$, and $\bar{\partial} f=0$ in $L^{1}(D)$. Therefore, $f$ is analytic in $D$.

The second question is: can one replace the set of polygons by other sets? This question is less simple. For example, one cannot replace polygons by the set $\sigma(B)$, where $B$ is a ball. Indeed, using the above argument one arrives at the relation $\int_{\sigma(B)} \bar{\partial} f d x d y=0$, and this does not imply that $\bar{\partial} f=0$, as the example on p. 2 shows. However, any domain $D$ which has $P$-property can be used in a generalization of the Morera theorem. By $\partial D$ the boundary of $D$ is denoted.

Theorem 4. Assume that $D$ has P-property, $f \in L_{l o c}^{1}\left(\mathbb{R}^{2}\right)$, and

$$
\int_{\partial \sigma(D)} f d z=0 \quad \forall \sigma \in G .
$$

Then $f$ is an entire function.

Proof. By the argument given above it is sufficient to prove this theorem assuming $f C^{1}-$ smooth. In this case one has

$$
\int_{\partial \sigma(D)} f d z=2 i \int_{\sigma(D)} \bar{\partial} f d x d y=0
$$

where $z=x+i y$. Since $D$ has $P$-property, one concludes from the above equation that $\bar{\partial} f=0$ in $\mathbb{R}^{2}$. This means that $f$ is an entire function. 
Let $B_{r}$ denote a ball (disc if $n=2$ ) of radius $r$ centered at the origin, $f \in L_{l o c}^{1}\left(\mathbb{R}^{2}\right)$, and $s_{j}, j=1,2, \ldots$, denote positive zeros of the Bessel function $J_{1}(s)$.

From here to the end of Section 3 it is assumed that $n=2$.

In [18] the following result is proved:

Lemma 4 If $\int_{\partial \sigma\left(B_{r}\right)} f d z=0$ for $r=r_{1}$ and for $r=r_{2}$, and if $r_{1} / r_{2}$ does not belong to the set $s_{j} / s_{m}$ for any positive integers $j$ and $m$, then $f$ is an entire function.

Proof. We give a new short proof of this result, showing that it is an immediate consequence of Theorem 4.

Indeed, it follows from Example 1 with $n=2$ that if (1) holds for $D=B_{r_{1}}$, then the support of $f$ belongs to the set $\mathcal{N}^{(1)}=\left\{\xi:|\xi|=s_{j} / r_{1}, \xi \in \mathbb{R}^{2}\right.$ for some positive integers $\left.j\right\}$, see the proof of Theorem 3 .

Similarly, if (1) holds for $D=B_{r_{2}}$, then the support of $f$ belongs to the set $\mathcal{N}^{(2)}=\left\{\xi:|\xi|=s_{m} / r_{2}, \xi \in\right.$ $\mathbb{R}^{2}$ for some positive integers $\left.m\right\}$. If these two sets, $\mathcal{N}^{(1)}$ and $\mathcal{N}^{(2)}$, have empty intersection, then the support of $f$ is empty, so that $f=0$. The role of $f$ will be played by $\bar{\partial} f$ in what follows.

The set $\mathcal{N}^{(1)}$ does not intersect the set $\mathcal{N}^{(2)}$ if and only if $s_{j} / r_{1} \neq s_{m} / r_{2}$ for any positive integers $j$ and $m$. This condition is equivalent to the condition that $r_{1} / r_{2}$ does not belong to the set $s_{j} / s_{m}$ for any positive integers $j$ and $m$. Under this condition one concludes that $\bar{\partial} f=0$, because the role of $f$ is played in our case by $\bar{\partial} f$, see equation (25). Thus, $f$ is an entire function. Lemma 4 is proved.

The proof of this result in [18] is much longer and more complicated.

\section{Another approach to Pompeiu problem and some remarks}

In Conjecture 3 we have used the equation

$$
\int_{D} e^{i k \alpha \cdot x} d x=0 \quad \forall \alpha \in S^{n-1}, \quad k=\text { const }>0 .
$$

In the derivation of this equation from equation (3), see Lemma 3, vector $\alpha$ can be an arbitrary complex vector $z \in M$, where $M \subset \mathbb{C}^{n}$ is an algebraic variety defined by the equation $z \cdot z=1$. Here $z \in \mathbb{C}^{n}$ and $z \cdot w:=\sum_{j=1}^{n} z_{j} w_{j}$. Note that $w_{j}$ is used in the definition of $z \cdot w$, and not its complex conjugate $\bar{w}_{j}$. Let $n=3$ and $z=a+i b$, where $a, b \in \mathbb{R}^{3}$. One checks easily that $z=a+i b \in M$ if and only if $a \cdot b=0$ and $a^{2}-b^{2}=1$, where $a^{2}:=a \cdot a$. Let $a=\left(\lambda^{2}+1\right)^{1 / 2}\left(e_{1} \cos \theta+e_{2} \sin \theta\right)$, where $e_{j}, j=1,2,3$, are unit vectors of a Cartesian basis in $\mathbb{R}^{3}$, and $\theta \in[0,2 \pi)$. Let $b=\lambda e_{3}$. Here $\lambda \in \mathbb{R}$ is an arbitrary number. One can easily check that $\pm a \pm i b \in M$.

Let us prove the following lemma:

Lemma 5. If (26) holds for all $\alpha \in S^{n-1}$ then it holds for all $\alpha \in M$.

Proof. For simplicity and without loss of generality the proof is given for $n=2$. The function $\tilde{\chi}(\xi)$ is an entire function of $\xi \in \mathbb{C}^{2}$. Let $\xi=\left(z_{1}, z_{2}\right), z_{j} \in \mathbb{C}, j=1,2, z_{j}=x_{j}+i y_{j}$. Assume that $\tilde{\chi}\left(x_{1}, x_{2}\right)=0$ if $x_{1}^{2}+x_{2}^{2}=1$. The function $g\left(z_{1}\right):=\tilde{\chi}\left(z_{1}, \sqrt{1-z_{1}^{2}}\right)$ is an analytic function of $z_{1}$ for $\left|z_{1}\right|<\delta$, where $\delta<1$. By our assumption, $g\left(x_{1}\right)=0$. By the uniqueness theorem for analytic functions of one complex variable, it follows that $g\left(z_{1}\right)=0$ for $\left|z_{1}\right|<\delta$, and, consequently, everywhere in the set $\left(z_{1}, \sqrt{1-z_{1}^{2}}\right)$, where $z_{1}$ runs through a set in $\mathbb{C}$ for which $\sqrt{1-z_{1}^{2}}$ is analytic. The union of this set and the set $\left(z_{1},-\sqrt{1-z_{1}^{2}}\right)$ is the variety $M$. So, if $\tilde{\chi}\left(x_{1}, x_{2}\right)=0$ in the set $x_{1}^{2}+x_{2}^{2}=1$, then $\tilde{\chi}\left(z_{1}, z_{2}\right)=0$ in $M$. Lemma 5 is proved.

Let us write equation (26) with $z=a \pm i b$ as

$$
\int_{D} e^{ \pm \lambda k x_{3}+i k\left(\lambda^{2}+1\right)^{1 / 2}\left(x_{1} \cos \theta+x_{2} \sin \theta\right)} d x_{1} d x_{2} d x_{3}=0 .
$$

Assume that $D \subset \mathbb{R}^{3}$ is a bounded domain $C^{1}$-diffeomorphic to a ball.

Conjecture 6. Under these assumptions equation (27) holds for all $\lambda \in \mathbb{R}^{1}$ and all $\theta \in[0,2 \pi)$ if and only if $D$ is a ball.

In order to prove Conjecture 6 it may help to assume additionally that $D$ is a convex centrally symmetric domain. Conjecture 6 is valid without additional assumptions: its particular case is Conjecture 3, which is obtained if $z \in S^{2}$, and the author thinks that Conjecture 3 is correct.

If $D$ is a ball of radius $R$, then equation (27) holds if $k R=s_{\frac{3}{2}, j}$, where $s_{\frac{3}{2}, j}$ are positive zeros of the Bessel function $J_{3 / 2}$. This follows from the calculations given in Example 1. Conjecture 6, if it is correct, says that equation (27) cannot hold for any domain, satisfying the stated assumptions, except for a ball.

We have formulated Conjecture 6 in the hope that a study of the asymptotic behavior of the integral (27) as $\lambda \rightarrow \infty$ may help to prove that the integral in (27) can be zero only if $D$ is a ball. 
Remark 2. It is proved in [10] and in [12] that if $D_{1} \in P$ and $D_{2}$ is a "sufficiently close" to $D_{1}$, then $D_{2} \in P$. This means that $P$-property is stable in some sense. The $\bar{P}$-property is not stable: small perturbations of $D$ lead to domains the Fourier transform of the characteristic function of which do not have a spherical surface of zeros.

The notion of being "sufficiently close" is defined as follows. The domain $D_{2}$ is $C^{3}$-smooth, strictly convex, its Gaussian curvature is bounded from below by a positive constant, and meas $\left(D_{12} \backslash D^{12}\right)$ is sufficiently small. Here $D^{12}:=D_{1} \cap D_{2}$ and $D_{12}:=D_{1} \cup D_{2}$.

Remark 3. One can prove (see, for example, [12], p.412) that if $D \subset \mathbb{R}^{3}$ is a bounded strictly convex domain with a smooth boundary, and $\tilde{\chi}_{D}\left(t_{m} \alpha\right)=0$ for all $\alpha \in S^{2}$ and a sequence $t_{m} \rightarrow+\infty$, then $D$ is a ball.

Remark 4. It is easy to give examples of the domains $D$ which have $P$-property: any polygon has this property since it does not have a real-analytic boundary. An ellipsoid in $\mathbb{R}^{n}, n \geq 2$, has $P$-property unless it is a ball. This is easy to check by calculating the Fourier transform of the characteristic function of an ellipsoid and checking that this Fourier transform does not have a spherical set of zeros. If the equation of the ellipsoid $D$ is $\sum_{j=1}^{n} \frac{x_{j}^{2}}{a_{j}^{2}}=1$, then the Fourier transform of its characteristic function is

$$
\tilde{\chi}_{D}=(2 \pi)^{n / 2}\left(\sum_{j=1}^{n} \xi_{j}^{2} a_{j}^{2}\right)^{-\frac{n}{2}} J_{n / 2}\left(\left(\sum_{j=1}^{n} \xi_{j}^{2} a_{j}^{2}\right)^{1 / 2}\right) .
$$

This is calculated by making the change of variables $x_{j}^{\prime}=x_{j} / a_{j}$, which transforms the ellipsoid into the ball of radius 1 and the $\xi_{j}$ variable of the Fourier transform into $\xi_{j} a_{j}$, so that then the formula for the Fourier transform of the characteristic function of the ball can be used. This formula is given in Example 1.

Remark 5. One can construct $f \neq 0$ satisfying equation (1) for the domain $D$, which fails to have $P$-property, by the following method, see [12], p.406. Let $|\xi|=b$ be the spherical surface $S_{b}$ of zeros of the Fourier transform of the characteristic function of $D$. Take any function $A(\xi) \in L^{1}\left(S_{b}\right)$ and define $\tilde{f}(\xi)=A(\xi) \delta(|\xi|-b)$, where $\delta(|\xi|-b)$ is the delta-function supported on the sphere $S_{b}$. Then the inverse Fourier transform of $\tilde{f}$ is a function $f \neq 0$, which satisfies equation (1). Since $\tilde{f}$ has compact support, by the Paley-Wiener theorem the function $f$ is an entire function of $x$. For example, if $n=3$ and $A(\xi)=1$, then

$$
f(x)=\frac{1}{(2 \pi)^{3}} \int_{|\xi|=b} e^{-i \xi \cdot x} d \xi=(2 \pi)^{-\frac{3}{2}} b^{2} \frac{J_{1 / 2}(b|x|)}{\sqrt{b|x|}}
$$

where the known formula

$$
e^{i k \alpha \cdot x}=\sum_{\ell=0}^{\infty} 4 \pi i^{\ell} j_{\ell}(k|x|) Y_{\ell}(\alpha) \overline{Y_{\ell}\left(x^{0}\right)}
$$

was used. In this formula $k>0$ is a constant, $\alpha \in S^{2}$ is a unit vector, $S^{2}$ is the unit sphere in $\mathbb{R}^{3}, Y_{\ell}$ are the normalized in $L^{2}\left(S^{2}\right)$ spherical harmonics, $x^{0}:=x /|x|, j_{\ell}(r):=\sqrt{\frac{\pi}{2 r}} J_{\ell+\frac{1}{2}}(r)$, and over-line stand for complex conjugate. Substitute for $e^{-i \xi \cdot x}$ the above sum and use the orthogonality of the spherical harmonics on the unit sphere. Then only the term with $\ell=0$ survives the integration over the sphere $|\xi|=b$. This essentially yields the expression for $f(x)$.

Let us give an illustration to the method of this Section by considering the two-dimensional Pompeiu problem.

In polar coordinates $x=r \cos \phi e_{1}+r \sin \phi e_{2}$. If $\alpha=\cos \theta e_{1}+\sin \theta e_{2}$, then $\alpha \cdot x=r \cos (\phi-\theta)$ and $\alpha \in M$ for any complex $\theta$. Consequently, $i k \alpha \cdot x=r \psi$, where $\psi:=i k \cos (\phi-\theta)$. One has

$$
\int_{D} e^{i \xi \cdot x} d x=\int_{-\pi}^{\pi} d \phi \int_{0}^{f(\phi)} d r r e^{r \psi}=0, \quad \forall \theta \in[-\pi, \pi],
$$

where $\theta \in[-\pi, \pi]$ is arbitrary, since one can choose an arbitrary $\alpha$. Taking the integral over $r$ in $(28)$ one gets

$$
\int_{-\pi}^{\pi}\left[\frac{e^{f(\phi) \psi} f(\phi)}{\psi}-\frac{e^{f(\phi) \psi}-1}{\psi^{2}}\right] d \phi=0
$$

Changing the integration variable, $\phi-\theta=\nu$, and using $2 \pi$ periodicity of $f$, one gets from (29) the following formula valid for all $\theta \in[-\pi, \pi]$ :

$$
\int_{-\pi}^{\pi}\left[\frac{e^{f(\nu+\theta) \psi_{0}} f(\nu+\theta)}{\psi_{0}}-\frac{e^{f(\nu+\theta) \psi_{0}}-1}{\psi_{0}^{2}}\right] d \nu=0, \quad \psi_{0}:=\left.\psi\right|_{\theta=0} .
$$


Differentiating (30) with respect to $\theta$ one obtains

$$
\int_{-\pi}^{\pi} f(\nu+\theta) f^{\prime}(\nu+\theta) e^{f(\nu+\theta) \psi_{0}} d \nu=0 \quad \forall \theta \in[-\pi, \pi] .
$$

Returning to the integration variable $\phi=\nu+\theta$ in equation (31) one obtains the following equation:

$$
\int_{-\pi}^{\pi} f(\phi) f^{\prime}(\phi) e^{f(\phi) \psi} d \phi=0 \quad \forall \theta \in M, \quad \psi=i k \cos (\phi-\theta) .
$$

Now one can choose in the above equation $\cos \theta=-i s, \sin \theta=\sqrt{s^{2}+1}$, where $s \in \mathbb{R}$ is an arbitrary real number. Note that the pair $\left\{-i s, \sqrt{s^{2}+1}\right\}$ belongs to the two-dimensional variety $M$. Clearly, $f=c$ is a solution to equation (32) if $c=$ const $>0$. If one can prove that this is the only solution to equation (32), then the two-dimensional Pompeiu problem is solved. One may try to study the asymptotic behavior of the integral in $(32)$ as $s \rightarrow \infty$ in order to show that equation (32) has no other solutions other than $f=c$. The difficulty in this approach lies in the fact that the phase in equation (32) is complex-valued, so the known results on the asymptotic of the integrals with large parameter do not apply.

\section{References}

[1] A.D. Alexandrov, A characteristic property of spheres, Ann. Mat. Pura Appl. 58 (1962) 303-315.

[2] L. Brown, B. Schreiber, B. Taylor, Spectral synthesis and the Pompeiu problem, Ann. Inst. Fourier 23 (1973) $125-154$.

[3] L. Chakalov, Sur un probléme de D.Pompeiu, Godishnik Univ. Sofia, Fac. Phys-Math. 40 (1944) 1-14.

[4] T. Chatelain and A. Henrot, Some results about Schiffer's conjectures, Inverse Problems, 15 (1999) 647-658.

[5] B. Fuks, Theory of analytic functions of several variables. AMS, Providence RI, 1963.

[6] M. Hirsch, Differential topology. Springer-Verlag, New York, 1976.

[7] L.Hörmander, The analysis of linear partial differential operators I. Springer-Verlag, Berlin, 1983.

[8] T. Kobayashi, Asymptotic behavior of the null variety for a convex domain in a non-positively curved space form, $J$. Fac. Sci.Univ. Tokyo Sect IA Math.36 (1989) 389-478.

[9] D. Pompeiu, Sur une propriété intégrale des fonctions de deux variables reelles, Bull. Sci. Acad. Roy. Belgique 5 (1929) 265-269.

[10] A. G. Ramm, The Pompeiu problem, Applicable Analysis 64 (1997) 19-26.

[11] A. G. Ramm, Necessary and sufficient condition for a domain, which fails to have Pompeiu property, to be a ball, Journ. of Inverse and Ill-Posed Probl. 6 (1998) 165-171.

[12] A. G. Ramm, Inverse Problems. Springer, New York, 2005.

[13] A. G. Ramm, A symmetry problem, Annal. Polon. Math. 92 (2007) 49-54.

[14] A. G. Ramm, Symmetry problems 2, Annal. Polon. Math. 96 (2009) 61-64.

[15] A. G. Ramm, Symmetry problem, Proc. Amer. Math. Soc. 141 (2013) 515-521.

[16] S. Williams, Analyticity of the boundary for Lipschitz domains without Pompeiu property, Indiana Univ. Math. Journ. 30 (1981) $357-369$.

[17] L. Zalcman, A bibliographical survey of the Pompeiu Problem, in the book Approximation by solutions of partial differential equations. Edited by B.Fuglede. Kluwer Acad., Dordrecht, 1992, pp. 177-186.

[18] L. Zalcman, Analyticity and the Pompeiu problem, Arch. Rat. Mech. Anal. 47 (1972) 237-254. 